\title{
DIFFERENTIAL REPRESENTATION OF A SAMUELSON MODEL WITH A TELEGRAPH DRIFT
}

\author{
Sergei A. Melnyk - Anna A. Kharkhota*
}

\begin{abstract}
In this paper we construct a system of three stochastic differential equations, which has a solution composed of a generalized telegraph signal process and a basic process. This system enabled us to find the escape probability of the basic process from an interval through its endpoint.
\end{abstract}

\section{Introduction}

P. S a muelson in [4 proposed the geometric Brownian motion as a model for dynamics of a financial asset price. The Samuelson model is widely known and studied and it became one of the most important models of financial mathematics. However, due to its serious drawback, i.e., the constant drift and diffusion coefficients, various modified models have been introduced [5]. In this paper we will consider the modified model proposed by $\mathrm{Kh}$ ark h ot a [2]:

$$
S(t)=S_{0} e^{\int_{0}^{t} \eta_{\nu(s)} \mathrm{d} s-0.5 \sigma^{2} t+\sigma w(t)} .
$$

This model differs from the classic one by the increase coefficient $\eta_{\nu(t)}$, which is a telegraph signal process, not a constant. Thus, it is much more difficult to study. The properties of the modified model and parameter estimation have been studied in [3]. The classic Samuelson model is a stochastic process, which solves the linear Ito stochastic differential equation with constant coefficients. The dynamics of solutions of stochastic differential equations is studied using the powerful instrument based on the partial differential equations theory. Our objective is construction of a system of stochastic differential equations describing the dynamics of the process $S(t)$ and satisfying the conditions of the classic theory of stochastic differential equations (see, for example, [1]). Then we

(C) 2017 Mathematical Institute, Slovak Academy of Sciences.

2010 Mathematics Subject Classification: 60G10, 60H10.

Keywords: stochastic differential equation, generalized telegraph signal process.

* The corresponding author. 
will be able to apply the results given in [1] for processes, which are solutions of stochastic differential equations, and study the dynamics of the process $S(t)$.

\section{Model}

We consider some probability space $(\Omega, \mathfrak{F}, \mathbf{P})$. On this space we define the following variables:

- $\eta$ is a $\mathcal{N}(0,1)$-distributed random variable;

- $\left\{\eta_{k}\right\}_{k=0}^{+\infty}$ is a sequence of independent random variables generated by $\eta$;

- $\nu(t)$ is a Poisson process with parameter $\lambda>0$, independent from the defined sequence;

- $w(t)$ is a Wiener process, independent from the processes above.

We denote by $\mathcal{N}(0,1)$ a standard normal distribution, by $\mathcal{U}(a, b)$ a uniform distribution on the interval $(a ; b)$.

We will also use these notations:

- $\mathfrak{G} \subseteq \mathfrak{F}$ is the $\sigma$-algebra, such that $\eta_{k} \prec \mathfrak{G}, k=0,1, \ldots$

- $\left\{\mathfrak{V}_{t} \subseteq \mathfrak{F}, \forall t \geq 0\right\}$ is the filtration generated by the process $\nu(t)$;

- $\left\{\mathfrak{W}_{t} \subseteq \mathfrak{F}, \forall t \geq 0\right\}$ is the filtration generated by the process $w(t)$;

- $\mathfrak{F}_{t}=\mathfrak{G} \times \mathfrak{V}_{t} \times \mathfrak{W}_{t}, \forall t \geq 0$.

On the probability space $\left(\Omega, \mathfrak{F}, \mathbf{P},\left\{\mathfrak{F}_{t}, t \geq 0\right\}\right)$ we consider the stochastic process

$$
z(t)=z_{0}+\mu(t)-0.5 \sigma^{2} t+\sigma w(t), t \geq 0 .
$$

Here, $\mu(t)=\int_{0}^{t} \eta(s) \mathrm{d} s, \eta(s)=\eta_{\nu(s)}, \sigma \in \mathbb{R}_{+}^{1}, z_{0} \in \mathbb{R}^{1}$. The process $\eta(t)$ is known as the generalized telegraph signal. Let us call the process $z(t)$ by the basic process.

The process $S(t)$ is the generalized Samuelson model (the geometric Brownian motion). In the Samuelson model $\mu(t)=\mu t$, that is, the coefficient $\mu$ is supposed to be nonrandom and constant. In the model (11) the drift coefficient is represented by the generalized telegraph signal process. Thanks to this, the model (1) allows changes of a trend of financial asset price at random times.

One of important problems which appear when studying the dynamics of the stochastic process is defining or estimating escape probability, i.e., the probability that it will leave some domain through a specific part of its boundary. The escape probability is widely used to quantify dynamical behavior of the stochastic process. In our case, the expression (11) is inconvenient for solving this problem. But if we manage to represent the basic process in a form allowing to include it to the closed system of stochastic differential equations, 
the problem will be solved by common methods. Let us describe how we will realize it. According to [6], the two-component process $(z(t), \eta(t))$ is a homogeneous Markov process with a discrete component $\eta(t)$. Then, for this pair of stochastic processes one can construct a system of stochastic differential equations with Poisson and Wiener processes as noises. This will allow us to construct distributions of functionals of these processes, applying the results of [1]. In particular, we will be able to find the escape probability of the basic process from a given interval through its endpoint.

Thus, we have to solve the following problems:

Problem 1. Constructing a closed system of stochastic differential equations solved by the processes $z(t)$ and $\eta(t)$.

Problem 2. Defining the escape probability of the basic process from a given interval through its endpoint.

\section{Auxiliary results}

The results of this section are based on Lemmas 3.1, 3.2 of [7]. According to them, random variables

$$
\eta=\sqrt{-2 \ln \alpha} \sin (2 \pi \beta), \kappa=\sqrt{-2 \ln \alpha} \cos (2 \pi \beta)
$$

are independent $\mathcal{N}(0,1)$-distributed, if $\alpha$ and $\beta$ are independent $\mathcal{U}(0,1)$-distributed random variables. Obviously, $\eta \prec \mathfrak{G}, \kappa \prec \mathfrak{G}$, if $\alpha \prec \mathfrak{G}, \beta \prec \mathfrak{G}$. We will prove that the inverse construction is also possible: by the given independent Gaussian random variables $\eta$ and $\kappa$ one can construct the appropriate independent uniformly distributed random variables $\alpha$ and $\beta$, which provide that (2) holds.

Let us define

$$
\varphi(x, y)=\left\{\begin{aligned}
\operatorname{arctg} \frac{\mathrm{y}}{\mathrm{x}}, & x>0, y \geq 0, \\
2 \pi+\operatorname{arctg} \frac{\mathrm{y}}{\mathrm{x}}, & x>0, y<0, \\
\pi+\operatorname{arctg} \frac{\mathrm{y}}{\mathrm{x}}, & x<0, \\
\frac{\pi}{2}, & x=0, y>0, \\
\frac{3 \pi}{2}, & x=0, y<0, \\
0, & x=0, y=0 .
\end{aligned}\right.
$$

Formulas $r=\sqrt{x^{2}+y^{2}}$ and $\varphi(x, y)$ express polar coordinates $r, \varphi$ through Cartesian coordinates $x, y$.

LEMма 1. If $\eta$ and $\kappa$ are independent $\mathcal{N}(0,1)$-distributed random variables, then the variable $\varphi(\eta, \kappa)$ is $\mathcal{U}(0,2 \pi)$-distributed. 
Proof. First, we notice that

$$
0 \leq \varphi(x, y) \leq 2 \pi, \quad \text { for all } \quad(x, y) \in \mathbb{R}^{2} .
$$

Let us construct a density function of the variable $\varphi(\eta, \kappa)$. We have

$$
\mathbb{P}\{\varphi(\eta, \kappa)<z\}=\mathbf{P}_{1}(z)+\mathbf{P}_{2}(z)+\mathbf{P}_{3}(z),
$$

where

$$
\begin{aligned}
& \mathbf{P}_{1}(z)=\mathbb{P}\{(\varphi(\eta, \kappa)<z) \cap(\eta>0) \cap(\kappa>0)\}, \\
& \mathbf{P}_{2}(z)=\mathbb{P}\{(\varphi(\eta, \kappa)<z) \cap(\eta>0) \cap(\kappa<0)\}, \\
& \mathbf{P}_{3}(z)=\mathbb{P}\{(\varphi(\eta, \kappa)<z) \cap(\eta<0)\} .
\end{aligned}
$$

From (4) we deduce that $\mathbb{P}\{\varphi(\eta, \kappa)<z\}=0$, if $z \leq 0$ and $\mathbb{P}\{\varphi(\eta, \kappa)<z\}=1$, if $z \geq 2 \pi$.

Let us consider the case of $0<z<2 \pi$. From (5) we get an expression for the density function of the variable $\varphi(\eta, \kappa)$ :

$$
\mathbf{p}(z)=\frac{d \mathbf{P}(z)}{d z}=\frac{d \mathbf{P}_{1}(z)}{d z}+\frac{d \mathbf{P}_{2}(z)}{d z}+\frac{d \mathbf{P}_{3}(z)}{d z}=\mathbf{p}_{1}(z)+\mathbf{p}_{2}(z)+\mathbf{p}_{3}(z) .
$$

We have to compute every summand. If $z \leq 0$, then $\mathbf{P}_{1}(z)=0$, and $\mathbf{P}_{1}(z)=1$, if $z \geq 0.5 \pi$. Let $z \in(0,0.5 \pi)$. Then we have

$$
\begin{aligned}
\mathbf{P}_{1}(z) & =\mathbb{P}\{(\kappa<\eta \operatorname{tgz}) \cap(\eta>0) \cap(\kappa>0)\} \\
& =\frac{1}{2 \pi} \int_{0}^{+\infty} e^{-0.5 x^{2}} \int_{0}^{x \operatorname{tgz}} e^{-0.5 y^{2}} \mathrm{~d} y \mathrm{~d} x .
\end{aligned}
$$

Hence

$$
\mathbf{p}_{1}(z)=\frac{1}{2 \pi \cos ^{2} z} \int_{0}^{+\infty} x e^{-0.5\left(1+\operatorname{tg}^{2} z\right) \mathrm{x}^{2}} \mathrm{~d} x=\frac{1}{2 \pi} \text {. }
$$

So, we get

$$
\mathbf{p}_{1}(z)= \begin{cases}0, & z \notin(0,0.5 \pi) \\ \frac{1}{2 \pi}, & z \in(0,0.5 \pi)\end{cases}
$$

Now let us find $p_{2}(z)$. We have

$$
\mathbf{P}_{2}(z)=\mathbb{P}\left\{\left(\operatorname{arctg} \frac{\kappa}{\eta}<z-2 \pi\right) \cap(\eta>0) \cap(\kappa<0)\right\} .
$$

If $\eta>0$ and $\kappa<0$, then $\operatorname{arctg} \kappa / \eta \in(-0.5 \pi, 0)$. Hence, $\mathbf{P}_{2}(z)=0$ if $z \leq 1.5 \pi$, and $\mathbf{P}_{2}(z)=1$ if $z \geq 2 \pi$. 
Let $z \in(1.5 \pi, 2 \pi)$. Then

$$
\mathbf{P}_{2}(z)=\frac{1}{2 \pi} \int_{0}^{+\infty} \mathrm{d} x \int_{-\infty}^{x \operatorname{tgz}} e^{-0.5\left(x^{2}+y^{2}\right)} \mathrm{d} y
$$

and

$$
\mathbf{p}_{2}(z)=\left\{\begin{aligned}
0, & z \notin(1.5 \pi, 2 \pi) \\
\frac{1}{2 \pi}, & z \in(1.5 \pi, 2 \pi)
\end{aligned}\right.
$$

Finally, we find $p_{3}(z)$. We have

$$
\mathbf{P}_{3}(z)=\mathbb{P}\left\{\left(\pi+\operatorname{arctg} \frac{\kappa}{\eta}<\mathrm{z}-2 \pi\right) \cap(\eta<0)\right\} .
$$

If $z \in[0,0.5 \pi]$, then $\mathbf{P}_{3}(z)=0$ and if $z \in[1.5 \pi, 2 \pi]$, then $\mathbf{P}_{2}(z)=1$. Let $z \in(0.5 \pi, 1.5 \pi)$. Then

$$
\mathbf{P}_{3}(z)=\mathbb{P}\{(\kappa>\eta \operatorname{tg} z) \cap(\eta<0)\}=\frac{1}{2 \pi} \int_{-\infty}^{0} e^{-0.5 x^{2}} \int_{x \operatorname{tgz}}^{+\infty} e^{-0.5 y^{2}} \mathrm{~d} y \mathrm{~d} x .
$$

Hence

$$
\mathbf{p}_{3}(z)=\left\{\begin{array}{cc}
0, & z \notin(0.5 \pi, 1.5 \pi), \\
\frac{1}{2 \pi}, & z \in(0.5 \pi, 1.5 \pi) .
\end{array}\right.
$$

Applying (7), (8), (9) to (6) gives:

$$
\mathbf{p}(z)=\left\{\begin{array}{cc}
0, & z \notin(0,2 \pi) \\
\frac{1}{2 \pi}, & z \in(0,2 \pi)
\end{array}\right.
$$

Lemma 1 is proved.

LEMmA 2. Let $\eta \prec \mathfrak{G}, \quad \kappa \prec \mathfrak{G}$ be independent $\mathcal{N}(0,1)$-distributed random variables. There exist independent $\mathcal{U}(0,1)$-distributed random variables $\alpha \prec \mathfrak{G}$ and $\beta \prec \mathfrak{G}$ such that equalities (2) hold.

P r o of. Let us change to polar coordinates: $\eta=r \cos \psi, \kappa=r \sin \psi, r \geq 0$, $\psi \in[0,2 \pi)$. Then, a variable $r^{2}=\eta^{2}+\kappa^{2}$ is exponentially distributed with parameter 0.5 and its distribution function is as follows

$$
F_{r^{2}}(x)=\left\{\begin{aligned}
0, & x \leq 0, \\
1-e^{-0.5 x}, & x>0 .
\end{aligned}\right.
$$

According to Smirnov theorem (see, for instance, [7]), a random variable

$$
\alpha=1-F_{r^{2}}\left(r^{2}\right)=e^{-0.5 r^{2}}=e^{-0.5\left(\eta^{2}+\kappa^{2}\right)}
$$

is $\mathcal{U}(0,1)$-distributed. So, we have $r=\sqrt{-2 \ln \alpha}$.

From Lemma 1. $\psi=\psi(\eta, \kappa)$ is $\mathcal{U}(0,2 \pi)$-distributed. Hence, $\beta=\frac{\psi}{2 \pi}$ is $\mathcal{U}(0,1)$ -distributed. So, we get equalities (2). 
Now, we will prove that $\alpha$ and $\beta$ are independent. For this purpose it is enough to show that $\eta^{2}+\kappa^{2}$ and $\frac{\kappa}{\eta}$ are independent. Let $x>0, y \in \mathbb{R}^{1}$. We have

$$
\begin{aligned}
\mathbb{P}\left\{\eta^{2}+\kappa^{2}<x, \frac{\kappa}{\eta}<y\right\} & =\frac{1}{2 \pi} \int_{u^{2}+v^{2}<x, u / v<y} e^{-0.5\left(u^{2}+v^{2}\right)} \mathrm{d} u \mathrm{~d} v \\
& =\left(1-e^{-0.5 x}\right)\left(\frac{\operatorname{arctgy}}{\pi}+\frac{1}{2}\right) \\
& =\mathbb{P}\left\{\eta^{2}+\kappa^{2}<x\right\} \mathbb{P}\left\{\frac{\kappa}{\eta}<y\right\} .
\end{aligned}
$$

So, the variables $\eta^{2}+\kappa^{2}$ and $\frac{\kappa}{\eta}$ are independent. This implies that $\alpha$ and $\beta$ are independent too. Lemma 2 is proved.

Lemma 3. Let $\alpha \prec \mathfrak{G}$ and $\beta \prec \mathfrak{G}$ be independent $\mathcal{U}(0,1)$-distributed random variables. Then

$$
\begin{array}{ll}
\eta_{j}=\sqrt{-2 \ln \alpha} \cos (2 \pi(j+1) \beta), & j=0,1, \ldots, \\
\kappa_{j}=\sqrt{-2 \ln \alpha} \sin (2 \pi(j+1) \beta), & j=0,1, \ldots
\end{array}
$$

are independent sequences of independent $\mathcal{N}(0,1)$-distributed random variables, such that $\eta_{0}=\eta, \kappa_{0}=\kappa, \eta_{j} \prec \mathfrak{G}, \kappa_{j} \prec \mathfrak{G}, j=0,1, \ldots$

Proof. Let us begin with proving that terms of the sequence are Gaussian random variables. We will do this by applying a method given in [7. Below we give some necessary results.

Definition 3.1. A random vector $\vec{\vartheta}=\left(\vartheta_{1}, \ldots, \vartheta_{n}\right)$ is called isotropic, if a point $\frac{\vec{\vartheta}}{|\vec{\vartheta}|}$ is uniformly distributed on a sphere $|\vec{\vartheta}|=1$ and is independent from $|\vec{\vartheta}|$.

LEMma 3.1. If $\vec{\vartheta}$ is an isotropic vector, such that $|\vec{\vartheta}|$ has $\chi_{n}$-distribution, then the components of this vector are independent $\mathcal{N}(0,1)$-distributed random variables.

Proof. Let us first prove that the vector $\vec{\vartheta}=\left(\eta_{j}, \kappa_{j}\right)$ is normally distributed for all $j=0,1, \ldots$ First we will prove that $|\vec{\vartheta}|$ has $\chi_{2}$-distribution. We have $|\vec{\vartheta}|=\sqrt{-2 \ln \alpha}$. Let $x>0$. Then

$$
\mathbb{P}\{|\vec{\vartheta}|<x\}=\mathbb{P}\left\{\alpha>e^{-0.5 x^{2}}\right\}=1-e^{-0.5 x^{2}}
$$

which is the $\chi_{2}$-distribution function. 
Let us now show that the vector $\frac{\vec{\vartheta}}{|\vec{\vartheta}|}$ is uniformly distributed on a unit circle. Clearly, the values of this vector lie on the unit circle. Let $l$ be some arc of this circle, limited by angles $0<\psi_{1}<\psi_{2}<2 \pi$. Since

$$
\left\{\frac{\vec{\vartheta}}{|\vec{\vartheta}|} \in l\right\}=\bigcup_{m=0}^{j}\left\{\frac{1}{j+1}\left(\frac{\psi_{1}}{2 \pi}+m\right)<\beta<\frac{1}{j+1}\left(\frac{\psi_{2}}{2 \pi}+m\right)\right\}
$$

we deduce that

$$
\mathbb{P}\left\{\frac{\vec{\vartheta}}{|\vec{\vartheta}|} \in l\right\}=\frac{\psi_{2}-\psi_{1}}{2 \pi}
$$

so, the vector $\frac{\vec{\vartheta}}{|\vec{\vartheta}|}$ is uniformly distributed on the unit circle.

Independence of $\frac{\vec{\vartheta}}{|\vec{\vartheta}|}$ and $|\vec{\vartheta}|$ easily follows from the expressions $|\vec{\vartheta}|=\sqrt{-2 \ln \alpha}$ and $\frac{\vec{\vartheta}}{|\vec{\vartheta}|}=(\cos (2 \pi(j+1) \beta), \sin (2 \pi(j+1) \beta))$, since $\alpha$ and $\beta$ are independent.

Thus, Lemma 3.1 is proved, i.e., the vector $\vec{\vartheta}=\left(\eta_{j}, \kappa_{j}\right)$ is normally distributed for all $j=0,1, \ldots$

To complete the proof of Lemma 3, we need to prove the independence of variables from (10). It is enough to show their uncorrelatedness. We have

$$
\mathbb{E} \eta_{j} \eta_{m}=-2 \mathbb{E} \ln \alpha \mathbb{E} \cos (2 \pi(j+1) \beta) \cos (2 \pi(m+1) \beta) .
$$

Since

$$
\mathbb{E} \ln \alpha=-1 \quad \text { and } \quad 2 \cos A \cos B=\cos (A+B)+\cos (A-B),
$$

then $2 \cos (2 \pi(j+1) \beta) \cos (2 \pi(m+1) \beta)=\cos (2 \pi(j-m) \beta)+\cos (2 \pi(j+m+2) \beta)$. If we notice that $j-m \neq 0, j+m+2 \neq 0$, then for all $s \in \mathbb{N}$ we have $\mathbb{E} \cos (2 \pi s \beta)=0$. Hence, $\mathbb{E} \cos (2 \pi(j+1) \beta) \cos (2 \pi(m+1) \beta)=0$ and $\mathbb{E} \eta_{j} \eta_{m}=0$, so, $\eta_{j}$ and $\eta_{m}$ are independent.

From

$$
\begin{aligned}
\mathbb{E} \kappa_{j} \kappa_{m} & =-2 \mathbb{E} \ln \alpha \mathbb{E} \sin (2 \pi(j+1) \beta) \sin (2 \pi(m+1) \beta) \\
& =\mathbb{E} \cos (2 \pi(j-m) \beta)-\mathbb{E} \cos (2 \pi(j+m+2) \beta)=0
\end{aligned}
$$

we deduce that $\kappa_{j}$ and $\kappa_{m}$ are independent. Finally, let us show the independence of $\eta_{j}$ and $\kappa_{m}$. We have

$$
\begin{aligned}
\mathbb{E} \eta_{j} \kappa_{m} & =-2 \mathbb{E} \ln \alpha \mathbb{E} \cos (2 \pi(j+1) \beta) \sin (2 \pi(m+1) \beta) \\
& =\mathbb{E} \sin (2 \pi(j-m) \beta)+\mathbb{E} \sin (2 \pi(j+m+2) \beta) .
\end{aligned}
$$


Let us compute $\mathbb{E} \sin (2 \pi s \beta), s=0,1, \ldots$ If $s=0$, then $\mathbb{E} \sin (2 \pi s \beta)=0$. Let $s \neq 0$. Then

$$
\mathbb{E} \sin (2 \pi s \beta)=\int_{0}^{1} \sin (2 \pi s x) d x=0 .
$$

Thus, $\mathbb{E} \eta_{j} \kappa_{m}=0$ for all integer non-negative $j$ and $m$. This implies that the random variables $\eta_{j}$ and $\kappa_{m}$ are independent. Lemma 3 is proved.

\section{Constructing the system of stochastic differential equations}

From now on we will consider Problem 1. A major attention is focused on the basic process $z(t)$, whose values we can observe experimentally. The dynamics of this process is described by the equation (11). The main disadvantage of this equation is its drift coefficient composing the stochastic process $\eta(t)$. It makes the study of the basic process much more complicated. However, this difficulty can be overcome by constructing a stochastic equation solved by the process $\eta(t)$. Let us define processes $\eta(t)$ and $\kappa(t)$ by equalities:

$$
\begin{aligned}
& \eta(t)=\sqrt{-2 \ln \alpha} \cos (2 \pi(\nu(t)+1) \beta), \\
& \kappa(t)=\sqrt{-2 \ln \alpha} \sin (2 \pi(\nu(t)+1) \beta), \quad t \geq 0 .
\end{aligned}
$$

Here, $\alpha \prec \mathfrak{G}, \beta \prec \mathfrak{G}$ are independent $\mathcal{U}(0,1)$-distributed random variables. By Lemma3, $\eta(t)$ and $\kappa(t)$ represent two independent generalized telegraph signal processes. Besides, $\eta(t) \prec \mathfrak{F}_{t}$ and $\kappa(t) \prec \mathfrak{F}_{t}, \forall t \geq 0$. Functions $\cos x$ and $\sin x$ are infinitely differentiable and their derivatives are bounded. Therefore, Theorem 1 from $\S 6$ of [1] applies and

$$
\begin{aligned}
d \eta(t)= & \lambda \sqrt{-2 \ln \alpha}[\cos (2 \pi \beta(\nu(t)+2))-\cos (2 \pi \beta(\nu(t)+1))] \mathrm{d} t \\
& +\sqrt{-2 \ln \alpha}[\cos (2 \pi \beta(\nu(t-)+2))-\cos (2 \pi \beta(\nu(t-)+1))] \tilde{\nu}(\mathrm{d} t) .
\end{aligned}
$$

Here we have used the equality $d \nu(t)=\lambda d t+\tilde{\nu}(d t)$, where $\tilde{\nu}(t)=\nu(t)-\lambda t$ is a centered Poisson process. We remembered that the average number of jumps of the Poisson process for the period $(0, t)$ is $E \nu(A, t)=\Pi(A) t$, where $\Pi(A)$ is a measure on some space $(X, \mathfrak{M})$ and $A \in \mathfrak{M}$. As we consider the standard Poisson process with unit jumps, we have $\Pi(\{1\})=\lambda$. Thus, integrals over a set of possible jump sizes of the process $\nu(t)$ equal to integrand values at $y=1$. 
By using the equalities $\cos (A+B)=\cos A \cos B-\sin A \sin B$ and (11), we get:

$$
\begin{aligned}
\cos (2 \pi \beta(\nu(t)+2))= & \cos 2 \pi \beta \cos (2 \pi \beta(\nu(t)+1)) \\
& -\sin 2 \pi \beta \sin (2 \pi \beta(\nu(t)+1))
\end{aligned}
$$

and

$$
\begin{gathered}
d \eta(t)=\lambda[(\cos (2 \pi \beta)-1) \eta(t)-\sin (2 \pi \beta) \kappa(t)] \mathrm{d} t \\
+[(\cos (2 \pi \beta)-1) \eta(t-)-\sin (2 \pi \beta) \kappa(t-)] \tilde{\nu}(\mathrm{d} t) \\
\eta(0)=\eta_{0}, \quad t \geq 0 .
\end{gathered}
$$

From (10) we deduce

$$
\begin{aligned}
& \cos (2 \pi \beta)=\frac{\eta_{0}}{\sqrt{\eta_{0}^{2}+\kappa_{0}^{2}}} \prec \mathfrak{F}_{0}, \\
& \sin (2 \pi \beta)=\frac{\kappa_{0}}{\sqrt{\eta_{0}^{2}+\kappa_{0}^{2}}} \prec \mathfrak{F}_{0} .
\end{aligned}
$$

Hence

$$
\begin{aligned}
\eta(t)=\eta_{0} & +\lambda \int_{0}^{t}\left[\left(\frac{\eta_{0}}{\sqrt{\eta_{0}^{2}+\kappa_{0}^{2}}}-1\right) \eta(s)-\frac{\kappa_{0}}{\sqrt{\eta_{0}^{2}+\kappa_{0}^{2}}} \kappa(s)\right] \mathrm{d} s \\
& +\int_{0}^{t}\left[\left(\frac{\eta_{0}}{\sqrt{\eta_{0}^{2}+\kappa_{0}^{2}}}-1\right) \eta(s-)-\frac{\kappa_{0}}{\sqrt{\eta_{0}^{2}+\kappa_{0}^{2}}} \kappa(s-)\right] \tilde{\nu}(\mathrm{d} s) .
\end{aligned}
$$

Similarly, we find the equation for the process $\kappa(t)$ :

$$
\begin{aligned}
\kappa(t)=\kappa_{0}+\lambda \int_{0}^{t}\left[\left(\frac{\eta_{0}}{\sqrt{\eta_{0}^{2}+\kappa_{0}^{2}}}-1\right) \kappa(s)+\frac{\kappa_{0}}{\sqrt{\eta_{0}^{2}+\kappa_{0}^{2}}} \eta(s)\right] \mathrm{d} s \\
+\int_{0}^{t}\left[\left(\frac{\eta_{0}}{\sqrt{\eta_{0}^{2}+\kappa_{0}^{2}}}-1\right) \kappa(s-)+\frac{\kappa_{0}}{\sqrt{\eta_{0}^{2}+\kappa_{0}^{2}}} \eta(s-)\right] \tilde{\nu}(\mathrm{d} s) .
\end{aligned}
$$

So, we get the result: 
TheOREM 1. Three processes: $z(t)$, defined by (1), $\eta(t)$ and $\kappa(t)$, both defined by (11), represent a solution for a closed system of stochastic differential equations:

$$
\left\{\begin{aligned}
z(t)=z_{0} & +\int_{0}^{t} \eta(s) \mathrm{d} s-0.5 \sigma^{2} t+\sigma w(t), \\
\eta(t)=\eta_{0}+ & \lambda \int_{0}^{t}\left[\left(\frac{\eta_{0}}{\sqrt{\eta_{0}^{2}+\kappa_{0}^{2}}}-1\right) \eta(s)-\frac{\kappa_{0}}{\sqrt{\eta_{0}^{2}+\kappa_{0}^{2}}} \kappa(s)\right] \mathrm{d} s \\
& +\int_{0}^{t}\left[\left(\frac{\eta_{0}}{\sqrt{\eta_{0}^{2}+\kappa_{0}^{2}}}-1\right) \eta(s-)-\frac{\kappa_{0}}{\sqrt{\eta_{0}^{2}+\kappa_{0}^{2}}} \kappa(s-)\right] \tilde{\nu}(\mathrm{d} s), \\
\kappa(t)=\kappa_{0} & +\lambda \int_{0}^{t}\left[\left(\frac{\eta_{0}}{\sqrt{\eta_{0}^{2}+\kappa_{0}^{2}}}-1\right) \kappa(s)+\frac{\kappa_{0}}{\sqrt{\eta_{0}^{2}+\kappa_{0}^{2}}} \eta(s)\right] \mathrm{d} s \\
& +\int_{0}^{t}\left[\left(\frac{\eta_{0}}{\sqrt{\eta_{0}^{2}+\kappa_{0}^{2}}}-1\right) \kappa(s-)+\frac{\kappa_{0}}{\sqrt{\eta_{0}^{2}+\kappa_{0}^{2}}} \eta(s-)\right] \tilde{\nu}(\mathrm{d} s),
\end{aligned}\right.
$$

where $z_{0} \in \mathbb{R}^{1}$ and $\eta_{0} \prec \mathfrak{F}_{0}, \kappa_{0} \prec \mathfrak{F}_{0}$ are independent $\mathcal{N}(0,1)$-distributed random variables.

Remark 1. If we notice that $\eta_{0} \prec \mathfrak{F}_{0}, \kappa_{0} \prec \mathfrak{F}_{0}, \eta(s) \prec \mathfrak{F}_{s}, \kappa(s) \prec \mathfrak{F}_{s}, \nu(s) \prec \mathfrak{F}_{s}$, it is clear that the stochastic integrals in (14) are well-defined.

Remark 2. The condition $\mathbb{E} \eta_{j}^{2}=1$ is not crucial. If $\mathbb{E} \eta_{j}^{2}=\sigma_{0}^{2}>0$, then it is sufficient just to change the first equation of the system (14) as follows:

$$
z(t)=z_{0}+\sigma_{0} \int_{0}^{t} \tilde{\eta}(s) \mathrm{d} s-0.5 \sigma^{2} t+\sigma w(t),
$$

where $\tilde{\eta}(s)=\eta(s) / \sigma_{0}$, and replace $\eta$ by $\tilde{\eta}$ everywhere.

The condition $\mathbb{E} \eta_{j}=0$ is not crucial, too. If $\mathbb{E} \eta_{j}=a$, then the first equation of (14) transforms to

$$
z(t)=z_{0}+\int_{0}^{t} \tilde{\eta}(s) \mathrm{d} s-0.5\left(\sigma^{2}-2 a\right) t+\sigma w(t),
$$

where $\tilde{\eta}(s)=\eta(s)-a$. 


\section{Escape probability of the basic process from a given interval through its right (left) endpoint}

In this section we will consider the Problem 2. Suppose the basic process is defined by the equation (11) and we need to find the escape probability from the interval $\left(z_{1}, z_{2}\right)$ through its right endpoint. The escape probability through the left endpoint can be found in a similar way, with the appropriate change of boundary conditions. Let $P(z, x, y)$ denote the probability that the vector process $(z(t), \eta(t), \kappa(t))$, starting at $(z, x, y)$, exits the region $G$, determined by $z_{1}<z<z_{2},-\infty<x<+\infty,-\infty<y<+\infty$ through the plain $z=z_{2}$. The system (14) of linear stochastic differential equations satisfies the conditions of Corollary 2 from $\S 11$ of [1]. Hence, $P(z, x, y)$ is a solution of the problem

$$
\begin{aligned}
& \frac{\partial^{2} P(z, x, y)}{\partial z^{2}}+\left(\frac{2 x}{\sigma^{2}}-1\right) \frac{\partial P(z, x, y)}{\partial z} \\
&+\frac{2 \lambda}{\sigma^{2}} P\left(z, \frac{x^{2}-y^{2}}{\sqrt{x^{2}+y^{2}}}, \frac{2 x y}{\sqrt{x^{2}+y^{2}}}\right)-\frac{2 \lambda}{\sigma^{2}} P(z, x, y)=0 \\
& P\left(z_{1}, x, y\right) \equiv 0, \quad P\left(z_{2}, x, y\right) \equiv 1, \quad z \in\left(z_{1}, z_{2}\right), \quad(x, y) \in \mathbb{R}^{2}
\end{aligned}
$$

This problem does not seem possible to be solved explicitly because of the third summand. However, to solve the Problem 2, we do not need $P(z, x, y)$, but

$$
\mathbb{P}\left\{z(\tau)=\left.z_{2}\right|_{z(0)=z}\right\}=\int_{-\infty}^{+\infty} \int_{-\infty}^{+\infty} P(z, x, y) \frac{1}{2 \pi} e^{-0.5\left(x^{2}+y^{2}\right)} \mathrm{d} x \mathrm{~d} y
$$

where $\tau$ is an escape moment of the process $z(t)$ from the interval $\left(z_{1}, z_{2}\right)$. Since $\eta(0)$ and $\kappa(0)$ are independent standard Gaussian random variables, (15) yields

$$
\begin{aligned}
& \int_{-\infty}^{+\infty} \int_{-\infty}^{+\infty}\left[\frac{\partial^{2} P(z, x, y)}{\partial z^{2}}+\left(\frac{2 x}{\sigma^{2}}-1\right) \frac{\partial P(z, x, y)}{\partial z}\right] \frac{1}{2 \pi} e^{-0.5\left(x^{2}+y^{2}\right)} \mathrm{d} x \mathrm{~d} y \\
& =\frac{2 \lambda}{\sigma^{2}} \int_{-\infty}^{+\infty} \int_{-\infty}^{+\infty} P(z, x, y) \frac{1}{2 \pi} e^{-0.5\left(x^{2}+y^{2}\right)} \mathrm{d} x \mathrm{~d} y \\
& -\frac{2 \lambda}{\sigma^{2}} \int_{-\infty}^{+\infty} \int_{-\infty}^{+\infty} P\left(z, \frac{x^{2}-y^{2}}{\sqrt{x^{2}+y^{2}}}, \frac{2 x y}{\sqrt{x^{2}+y^{2}}}\right) \frac{1}{2 \pi} e^{-0.5\left(x^{2}+y^{2}\right)} \mathrm{d} x \mathrm{~d} y .
\end{aligned}
$$


Let us transform the last summand in the right part of (16). By a change of variable

we obtain

$$
\frac{x^{2}-y^{2}}{\sqrt{x^{2}+y^{2}}}=u, \quad \frac{2 x y}{\sqrt{x^{2}+y^{2}}}=v
$$

$$
\begin{aligned}
& \qquad \begin{array}{l}
\int_{-\infty}^{+\infty} \int_{-\infty}^{+\infty} P\left(z, \frac{x^{2}-y^{2}}{\sqrt{x^{2}+y^{2}}}, \frac{2 x y}{\sqrt{x^{2}+y^{2}}}\right) \frac{1}{2 \pi} e^{-0.5\left(x^{2}+y^{2}\right)} \mathrm{d} x \mathrm{~d} y \\
=\frac{1}{2} \int_{-\infty}^{+\infty} \int_{-\infty}^{+\infty} P(z, u, v) \frac{1}{2 \pi} e^{-0.5\left(u^{2}+v^{2}\right)} \mathrm{d} u \mathrm{~d} v .
\end{array} \\
& \text { Substituting this in (16), we get }
\end{aligned}
$$

$$
\begin{aligned}
\int_{-\infty}^{+\infty} \int_{-\infty}^{+\infty}\left[\frac{\partial^{2} P(z, x, y)}{\partial z^{2}}+\left(\frac{2 x}{\sigma^{2}}-1\right)\right. & \frac{\partial P(z, x, y)}{\partial z} \\
& \left.-\frac{\lambda}{\sigma^{2}} P(z, x, y)\right] \frac{1}{2 \pi} e^{-0.5\left(x^{2}+y^{2}\right)} \mathrm{d} x \mathrm{~d} y=0 .
\end{aligned}
$$

Hence

$$
\begin{aligned}
& \mathbb{P}\left\{z(\tau)=\left.z_{2}\right|_{z(0)=z}\right\}=\int_{-\infty}^{+\infty} \int_{-\infty}^{+\infty} P(z, x, y) \frac{1}{2 \pi} e^{-0.5\left(x^{2}+y^{2}\right)} \mathrm{d} x \mathrm{~d} y \\
& \quad=\frac{\sigma^{2}}{\lambda} \int_{-\infty}^{+\infty} \int_{-\infty}^{+\infty}\left[\frac{\partial^{2} P(z, x, y)}{\partial z^{2}}+\left(\frac{2 x}{\sigma^{2}}-1\right) \frac{\partial P(z, x, y)}{\partial z}\right] \frac{1}{2 \pi} e^{-0.5\left(x^{2}+y^{2}\right)} \mathrm{d} x \mathrm{~d} y .
\end{aligned}
$$

Therefore, if we solve the problem

$$
\begin{array}{r}
\frac{\partial^{2} P(z, x, y)}{\partial z^{2}}+\left(\frac{2 x}{\sigma^{2}}-1\right) \frac{\partial P(z, x, y)}{\partial z}-\frac{\lambda}{\sigma^{2}} P(z, x, y)=0, \\
P\left(z_{1}, x, y\right) \equiv 0, \quad P\left(z_{2}, x, y\right) \equiv 1, \quad z \in\left(z_{1}, z_{2}\right), \quad(x, y) \in \mathbb{R}^{2},
\end{array}
$$

we will obtain the desired probability by integrating the solution with a corresponding weighting function.

The problem (18) can be solved in explicit form. Let us denote $\tilde{x}=\frac{2 x}{\sigma^{2}}-1$, $D=\tilde{x}^{2}+\frac{4 \lambda}{\sigma^{2}}$. The solution of (18) is

$$
P(z, x, y)=e^{0.5(\tilde{x}+\sqrt{D})\left(z_{2}-z\right)} \frac{e^{\sqrt{D}\left(z-z_{1}\right)}-1}{e^{\sqrt{D}\left(z_{2}-z_{1}\right)}-1} .
$$

Note that $0<P(z, x, y)<1, \forall z \in\left(z_{1}, z_{2}\right),(x, y) \in \mathbb{R}^{2}$. Indeed, $0<P(z, x, y)$ because $z_{1}<z<z_{2}$. Since $\tilde{x}<\sqrt{D}$, then (19) implies that

$$
P(z, x, y)<\frac{e^{\sqrt{D}\left(z_{2}-z_{1}\right)}-e^{\sqrt{D}\left(z_{2}-z\right)}}{e^{\sqrt{D}\left(z_{2}-z_{1}\right)}-1}<1 .
$$


Thus, the expression (19) is correct.

Besides,

$$
\begin{aligned}
\lim _{\lambda \rightarrow 0} P(z, x, y) & =\frac{1-e^{-\tilde{x}\left(z-z_{1}\right)}}{1-e^{-\tilde{x}\left(z_{2}-z_{1}\right)}}, \\
\lim _{\lambda \rightarrow+\infty} P(z, x, y) & =e^{0.5 \tilde{x}\left(z_{2}-z\right)} \lim _{\lambda \rightarrow+\infty} e^{-0.5\left(z_{2}-z\right) \sqrt{D}} \frac{1-e^{-\sqrt{D}\left(z-z_{1}\right)}}{1-e^{-\sqrt{D}\left(z_{2}-z_{1}\right)}}=0, \\
\lim _{z_{2} \rightarrow+\infty} P(z, x, y) & =0 .
\end{aligned}
$$

So, we get the solution of the Problem 2:

$$
\begin{aligned}
\mathbb{P}\left\{z(\tau)=\left.z_{2}\right|_{z(0)=z}\right\} & =\int_{-\infty}^{+\infty} \int_{-\infty}^{+\infty} P(z, x, y) \frac{1}{2 \pi} e^{-0.5\left(x^{2}+y^{2}\right)} \mathrm{d} x \mathrm{~d} y \\
& =\frac{1}{\sqrt{2 \pi}} \int_{-\infty}^{+\infty} e^{0.5(\tilde{x}+\sqrt{D})\left(z_{2}-z\right)} \frac{e^{\sqrt{D}\left(z-z_{1}\right)}-1}{e^{\sqrt{D}\left(z_{2}-z_{1}\right)}-1} e^{-\frac{x^{2}}{2}} \mathrm{~d} x .
\end{aligned}
$$

Let $Q(z, x, y)$ denote the escape probability that the vector process

$$
(z(t), \eta(t), \kappa(t)), \quad \text { starting at }(z, x, y),
$$

exits the region $G$ through the plane $z=z_{1}$. Then similar computations give

$$
Q(z, x, y)=e^{-0.5(\tilde{x}+\sqrt{D})\left(z-z_{1}\right)} \frac{e^{\sqrt{D}\left(z_{2}-z\right)}-1}{e^{\sqrt{D}\left(z_{2}-z_{1}\right)}-1}
$$

and

$$
\begin{aligned}
& \mathbb{P}\left\{z(\tau)=\left.z_{1}\right|_{z(0)=z}\right\} \\
& =\frac{1}{\sqrt{2 \pi}} \int_{-\infty}^{+\infty} e^{0.5(\tilde{x}+\sqrt{D})\left(z-z_{1}\right)} \frac{e^{\sqrt{D}\left(z_{2}-z\right)}-1}{e^{\sqrt{D}\left(z_{2}-z_{1}\right)}-1} e^{-0.5 x^{2}} \mathrm{~d} x .
\end{aligned}
$$

Since $\tilde{x}<\sqrt{D}$, then $P(z, x, y)+Q(z, x, y)<1$. It means, there is a positive probability that the process $(z(t), \eta(t), \kappa(t))$ will never escape from the region $G$ and so, the process $z(t)$ will not leave the interval $\left(z_{1}, z_{2}\right)$. In addition, we notice that

$$
\begin{aligned}
& \lim _{\lambda \rightarrow 0} Q(z, x, y)=1-\frac{1-e^{-\tilde{x}\left(z-z_{1}\right)}}{1-e^{-\tilde{x}\left(z_{2}-z_{1}\right)}}=1-\lim _{\lambda \rightarrow 0} P(z, x, y), \\
& \lim _{\lambda \rightarrow+\infty} Q(z, x, y)=e^{-0.5 \tilde{x}\left(z_{1}-z\right)} \lim _{\lambda \rightarrow+\infty} e^{-0.5\left(z-z_{1}\right) \sqrt{D}} \frac{1-e^{-\sqrt{D}\left(z_{2}-z\right)}}{1-e^{-\sqrt{D}\left(z_{2}-z_{1}\right)}}=0, \\
& \lim _{z_{2} \rightarrow+\infty} Q(z, x, y)=e^{-0.5(\tilde{x}+\sqrt{D})\left(z-z_{1}\right)} .
\end{aligned}
$$


Thus, we conclude that when the trend changes frequently ( $\lambda$ is large), the escape probability of the process $z(t)$ from the arbitrary interval $\left(z_{1}, z_{2}\right)$ becomes negligibly small, that is, the process stabilizes. On the other hand, if changes of the trend are rare, i.e., $\lambda$ is small, then the escape probability is close to 1 .

In addition to the obtained results, we will find mean residence time of the process in a given interval. Let $m(z, x, y)$ denote the mean residence time of the vector process $(z(t), \eta(t), \kappa(t))$ in the region $G$. By Corollary 1 from $\S 11$ of [1], $m(z, x, y)$ is the solution of the problem

$$
\begin{gathered}
\frac{\partial^{2} m(z, x, y)}{\partial z^{2}}+\tilde{x} \frac{\partial m(z, x, y)}{\partial z}-\frac{\lambda}{\sigma^{2}} m(z, x, y)=-1 \\
m\left(z_{1}, x, y\right) \equiv 0, \quad m\left(z_{2}, x, y\right) \equiv 0, \quad z \in\left(z_{1}, z_{2}\right), \quad(x, y) \in \mathbb{R}^{2} .
\end{gathered}
$$

Computing this solution, we get

$$
\begin{gathered}
m(z, x, y)=\frac{\sigma^{2}}{\lambda}\left[e^{-\sqrt{D}\left(z_{2}-z_{1}\right)+0.5(\tilde{x}+\sqrt{D})\left(z_{2}-z\right)} \frac{1-e^{-0.5(\tilde{x}-\sqrt{D})\left(z_{2}-z_{1}\right)}}{1-e^{-\sqrt{D}\left(z_{2}-z_{1}\right)}}\right. \\
\left.\quad-e^{0.5(\tilde{x}-\sqrt{D})\left(z_{2}-z\right)} \frac{1-e^{-0.5(\tilde{x}+\sqrt{D})\left(z_{2}-z_{1}\right)}}{1-e^{-\sqrt{D}\left(z_{2}-z_{1}\right)}}+1\right] .
\end{gathered}
$$

Correspondingly,

$$
m(z)=\mathbb{E}\left\{\left.\tau\right|_{z(0)=z}\right\}=\frac{1}{2 \pi} \int_{-\infty}^{+\infty} \int_{-\infty}^{+\infty} m(z, x, y) e^{-0.5\left(x^{2}+y^{2}\right)} \mathrm{d} x \mathrm{~d} y
$$

is the mean residence time of the process $z(t)$ in the interval $\left(z_{1}, z_{2}\right)$. Besides,

$$
\begin{aligned}
\lim _{\lambda \rightarrow 0} m(z, x, y) & =+\infty \\
\lim _{\lambda \rightarrow+\infty} m(z, x, y) & =0, \\
\lim _{z_{2} \rightarrow+\infty} m(z, x, y) & =\frac{\sigma^{2}}{\lambda}\left[1-e^{-0.5(\tilde{x}+\sqrt{D})\left(z-z_{1}\right)}\right] .
\end{aligned}
$$

The obtained results allow us to determine the dynamics of the process $S(t)$. For example, if $z_{1}=0, z_{2}=+\infty, z=z(0)=\ln S_{0}>0$, then we have

$$
\begin{aligned}
\mathbb{P}\left\{S(t)>S_{0}, \forall t \geq 0\right\} & =1-\mathbb{P}\left\{z(\tau)=\left.0\right|_{\left.z=\ln S_{0}\right\}}\right. \\
& =\frac{1}{\sqrt{2 \pi}} \int_{-\infty}^{+\infty} e^{-0.5 \ln S_{0}(\tilde{x}+\sqrt{D})} e^{-0.5 x^{2}} \mathrm{~d} x, \\
m\left(\ln S_{0}\right) & =\frac{\sigma^{2}}{\lambda}\left[1-\frac{1}{\sqrt{2 \pi}} \int_{-\infty}^{+\infty} S_{0}^{-0.5(\tilde{x}+\sqrt{D})} e^{-0.5 x^{2}} \mathrm{~d} x\right] .
\end{aligned}
$$


EXAmple 1. As an illustration let us consider a numerical example. Let

$$
\lambda=1, \quad \sigma^{2}=2, \quad z_{1}=0, \quad z=1, \quad z_{2}=+\infty .
$$

Then $\mathbb{P}\left\{z(\tau)=\left.0\right|_{z(0)=1}\right\} \approx 0.88, \mathbb{P}\left\{z(\tau)=+\left.\infty\right|_{z(0)=1}\right\}=0, m(1) \approx 0.69$. Thus, the probability that the process $z(t)$ will never leave the upper half plane (starting at $z(0)=1$ ) approximately equals to 0.12 . If $S(t)$ represents a financial asset price at time $t$, then the obtained values mean that the probability of price fall below the initial value is rather high (0.88), whereas mean time of the fall is rather small (0.69). Therefore, buying an asset at $t=0$ is risky and inefficient.

Remark 3. To solve the problems discussed, we have used the non-observable component $\eta(t)$ and auxiliary component $\kappa(t)$, which was not initially defined at all. However, thanks to the known distributions of the random variables $\eta(0)$ and $\kappa(0)$, we were able to average the solutions (19), (21), (23). As a result, the obtained expressions (20), (22) and (24) depend only on the parameters of the initial problem: $z, z_{1}, z_{2}, \lambda, \sigma_{0}, \sigma$.

\section{REFERENCES}

[1] GIKHMAN, I. I.-SKOROKHOD, A. V.: Stochastic Differential Equations. Kiev, Naukova Dumka, 1968.

[2] KHARKHOTA, A. A.: Properties of the Samuelson model with a telegraph drift, in: Proceedings of the IAMM of the NAS of Ukraine. 27 (2014), 217-225.

[3] KHARKHOTA, A. A.-MELNYK, S. A.: Estimation of parameters of the Samuelson model with telegraph drift, Ukr. Mat. Visn. 12 (2015), 557-572.

[4] SAMUELSON, P. A.: Rational theory of warrant pricing, Industrial Management Review 6 (1965), 13-31.

[5] SHIRYAEV, A. N.: Essentials of Stochastic Finance. Vol. 1. Facts. Models. Moscow, Fazis, 1998.

[6] SKOROKHOD, A. V.: Asymptotic Methods in the Theory of Stochastic Differential Equations. Kiev, Naukova Dumka, 1987.

[7] YASINSKA, L. I.-YASINSKIY, V.K.-YURCHENKO, I. V.: Simulation Modeling on Computer. Chernivtsi, Zelena Bukovyna, 1999.

Sergei A. Melnyk

V. I. Vernadsky Crimean

Federal University

Yalta

E-mail: s.a.melnik@yandex.ua

Anna A. Kharkhota

Institute of Applied Mathematics and Mechanics of the NAS of Ukraine

Slovyansk

UKRA ̈INE

E-mail: annaharhota@yandex.ru. 